\title{
Simulation Evaluation of Filtering Method for Improving Pedestrian Positioning Accuracy Using Signal Strengths
}

\author{
Yuya Nishimaki' ${ }^{1}$, Hisato Iwai ${ }^{2}$, Kenya Sato ${ }^{1}$ \\ ${ }^{1}$ Computer and Information Science, Graduate School of Science and Engineering, Doshisha University, Kyoto, Japan \\ ${ }^{2}$ Electrical and Electronic Engineering, Graduate School of Science and Engineering, Doshisha University, Kyoto, Japan \\ Email: yuya.nishimaki@nislab.doshisha.ac.jp, iwai@mail.doshisha.ac.jp, ksato@mail.doshisha.ac.jp
}

How to cite this paper: Nishimaki, Y., Iwai, H. and Sato, K. (2019) Simulation Evaluation of Filtering Method for Improving Pedestrian Positioning Accuracy Using Signal Strengths. Wireless Sensor Network, 11, 81-94.

https://doi.org/10.4236/wsn.2019.116006

Received: June 1, 2019

Accepted: June 25, 2019

Published: June 28, 2019

Copyright () 2019 by author(s) and Scientific Research Publishing Inc. This work is licensed under the Creative Commons Attribution International License (CC BY 4.0).

http://creativecommons.org/licenses/by/4.0/

c) (i) Open Access

\begin{abstract}
In recent years, we have been able to use various services using the position information of smartphones and tablets. In addition, research on intelligent transport systems (ITS) has been actively conducted. To consider reducing traffic accidents by exchanging position information between pedestrians and vehicles by vehicle-to-pedestrian communication, we require accurate position information for pedestrians and vehicles. The GPS (global positioning system) is the most widely used method for acquiring position information. However, in urban areas, the GPS signal is affected by the surrounding buildings, which increases the positioning error. In this study, a method to improve the positioning accuracy of pedestrians using the signal strengths from vehicles and beacons was proposed. First, a Kalman filter was applied to the signal strength. Then, the path loss index was dynamically calculated using vehicle-to-vehicle communication. Finally, the position of a pedestrian was obtained using weighted centroid localization (WCL) after filtering the nodes. The positioning accuracy was evaluated using a simulator and demonstrated the superiority of the proposed method.
\end{abstract}

\section{Keywords}

Outdoor Positioning, Vehicle-to-Pedestrian Communication,

Vehicle-to-Vehicle Communication, Signal Strength, Path Loss Index, WCL

\section{Introduction}

In recent years, intelligent transport systems (ITSs) have been actively researched for improving automobile safety and driver comfort. For example, vehicle-to-vehicle communication and vehicle-to-pedestrian communication ena- 
ble vehicles and pedestrians to exchange their information including their position and speed. There have been efforts to reduce traffic accidents by using such technology [1] [2]. To prevent accidents, the accuracy of each other's position information is important. A positioning system is a method to acquire position information, and the global positioning system (GPS) is the most commonly used. However, the positioning error is several meters to several tens of meters. In urban areas, GPS signals can be blocked by buildings and are affected by multipath, which increases the positioning error [3] [4]. As described above, since the accuracy of GPS is affected by the surrounding environment, it is difficult to estimate the position stably. Generally, pedestrians use only GPS to estimate their position, but in vehicles we can combine several methods, including not only GPS but also map matching and lane detection with in-vehicle cameras [5] [6]. As a result, a vehicle can estimate its position with higher accuracy than a pedestrian. Therefore, in this study, we focus on the positioning accuracy of pedestrians in urban areas and propose a method to improve the accuracy by using the signal strengths from vehicles and beacons.

The rest of this paper is organized as follows. Section 2 describes existing methods. Our proposed method is described in Section 3. The results of the simulation and evaluation are shown in Section 4. Finally, we summarize our paper in Section 5 .

\section{Existing Method}

\subsection{GPS}

GPS is the most commonly used positioning method. A device equipped with a GPS function estimates its position by receiving GPS signals transmitted from multiple satellites. In places where the line of sight is good and there are no tall buildings around, the positioning error is smaller because many GPS signals can be received. On the other hand, in places where there are many high-rise buildings in urban areas, GPS signals can be affected by blockage, reflection, and diffraction due to these high-rise buildings, and positioning errors can exceed 10 m.

\subsection{Positioning Method Using Signal Strength}

We can know the signal strength when a smartphone or tablet device receives signals emitted from a node such as a BLE (Bluetooth low energy) node or Wi-Fi node. It is possible to estimate the position of the device using these signal strengths [7] [8]. This method uses the characteristic that the signal strength attenuates with distance. When a device is communicating with several nodes, its position is estimated using their position information and the distances to each node obtained from signal strengths. In general, Equation (1) is used to obtain the distance from the signal strength.

$$
P(d)=10 n \log _{10} d
$$

Here, $P(d)$ is the signal strength at a position $d$ meters away from the sender, 
$A$ is the signal strength at a position 1 meters away from the sender, and $n$ is the path loss index. The value is $n=2$ in free space, but in fact it varies depending on the surrounding radio wave propagation environment. Therefore, this value should be given dynamically, but that is not done with existing methods. In addition, the signal strength varies due to fading and shadowing, and the fluctuation increases as the signal strength decreases. To reduce the distance error caused by fading and shadowing, using the average signal strength or applying filtering has been considered [7] [9].

There are several methods for obtaining the target position using the source position and distances estimated from the signal strengths. Now, there are $m(m \leq 3)$ available nodes whose positions are $\left(x_{1}, y_{1}\right),\left(x_{2}, y_{2}\right), \cdots,\left(x_{m}, y_{m}\right)$, and the distances from each node to the target are $d_{1}, d_{2}, \cdots, d_{m}$. In this situation, let us consider estimating position $(x, y)$ of the target. Equation (2) is obtained from the above situation. To determine the position of the target, it is linearized and then the least squares method is used [10].

$$
\left\{\begin{array}{c}
\left(x_{1}-x\right)^{2}+\left(y_{1}-y\right)^{2}=d_{1}^{2} \\
\left(x_{2}-x\right)^{2}+\left(y_{2}-y\right)^{2}=d_{2}^{2} \\
\vdots \\
\left(x_{m}-x\right)^{2}+\left(y_{m}-y\right)^{2}=d_{m}^{2}
\end{array}\right.
$$

The weighted centroid localization (WCL) is also used to determine the target position. WCL is a technique that uses a weighted average for positioning. The weight is often the inverse of the power of the distance [11] [12]. The target position is obtained by weighted averaging the $x$ and $y$ coordinates of each node as in Equation (3), Equation (4).

$$
\begin{gathered}
(x, y)=\left(\frac{\sum_{i=1}^{m} x_{i} w_{i}}{\sum_{i=1}^{m} w_{i}}, \frac{\sum_{i=1}^{m} y_{i} w_{i}}{\sum_{i=1}^{m} w_{i}}\right) \\
w_{i}=\frac{1}{d_{i}^{g}}
\end{gathered}
$$

\subsection{Positioning Using Vehicle Signal}

In the era of automatic driving, vehicles periodically transmit their presence and behavior to the neighboring vehicles and pedestrians to prevent traffic accidents or to automatically adjust the distance between vehicles to prevent or reduce traffic jams. That is, since the pedestrian can receive signals from the vehicle, the vehicle can also be treated as a node. Tang et al. estimated the position of pedestrians by combining GPS signals with vehicle signals [13]. Because signal strength has the above-mentioned problems, Tang et al. used channel state information instead of signal strength and used signals with line-of-sight path to determine pedestrian-vehicle distance with higher precision. Vehicle signals are also used in this study, which reduces the distance error and positioning error by approach different from the method in [13]. 


\section{Proposed Method}

\subsection{Outline}

In our proposed method, as shown in Figure 1, a pedestrian estimate his position using the signal strengths from beacons installed on the side of roads and the signal strengths from vehicles. It is assumed that vehicles periodically transmit their vehicle information to the surroundings. To estimate the position with high accuracy using the signal strength, it is necessary to accurately obtain the distance from the signal strength. However, as mentioned earlier, there are some problems with using the signal strength. Therefore, in our proposed method, filtering is applied to reduce the fluctuation of the signal strength. In addition, we use the dynamic path loss index calculated using vehicle-to-vehicle communication, which is used when calculating the pedestrian-vehicle distance. The pedestrian obtains his position using WCL after filtering the nodes, which copes with the increase in the number of surrounding nodes and the bias of the nodes.

\subsection{Filtering Signal Strength}

Our proposed method uses a Kalman filter that can perform filtering sequentially. The Kalman filter is applied to reduce the fluctuation of the signal strengths received by a pedestrian from vehicles and beacons. Filtering is performed using Equations (5) to (9).

$$
\begin{gathered}
\hat{x}^{-}(k)=\hat{x}(k-1) \\
\hat{P}^{-}(k)=\hat{P}(k-1)+Q \\
g(k)=\frac{\hat{P}^{-}(k)}{\hat{P}^{-}(k)+R} \\
\hat{x}(k)=\hat{x}^{-}(k)+g(k)\left(y(k)-\hat{x}^{-}(k)\right) \\
\hat{P}(k)=(1-g(k)) \hat{P}^{-}(k)
\end{gathered}
$$

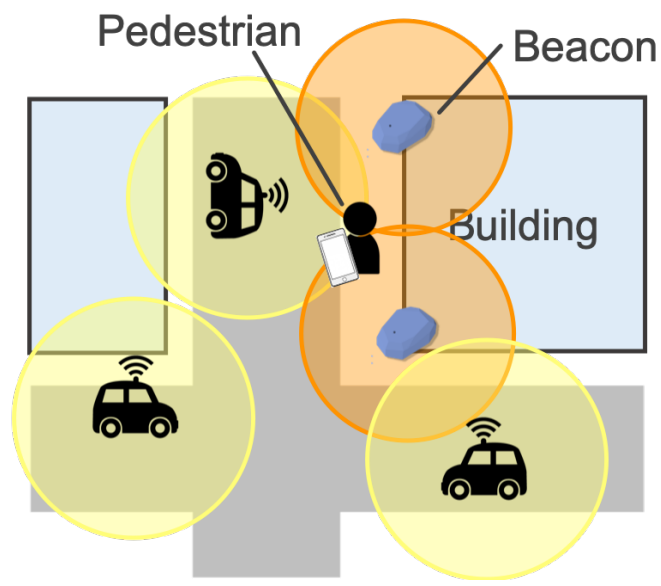

Figure 1. Outline of proposed method. 
Here, $\hat{x}^{-}(k)$ and $\hat{x}(k)$ are the prior and posteriori state estimate, respectively. Similarly, $\hat{P}^{-}(k)$ and $\hat{P}(k)$ are the prior and posteriori error variance, respectively, $y(k)$ is the measurement value, $g(k)$ is the Kalman gain, $Q$ is the system noise variance, and $R$ is the measurement noise variance. $\hat{P}(0), Q$ and $R$ were set to the values shown in Table 1 and Table 2 . We determined these values in advance using the maximum likelihood method based on values obtained by simulation.

\subsection{Calculation of Dynamic Path Loss Index Using Vehicle-to-Vehicle Communication}

When vehicle $k$ performs vehicle-to-vehicle communication with $m$ surrounding vehicles, the path loss index between vehicle $i(1 \leq i \leq m)$ and vehicle $k$ can be obtained from Equation (10), which is a modification of Equation (1). Here, $d_{i}$ is the Euclidean distance between vehicle $i$ and vehicle $k$, and $P\left(d_{i}\right)$ is the signal strength from vehicle $i$ received by vehicle $k$. To obtain the path loss index within a certain range, we set threshold $P$ th and use signal strengths satisfying $P\left(d_{i}\right)>P$ th. Then, Equation (11) calculates the average of the path loss index of the surrounding vehicles obtained within a certain time. The result is the path loss index around vehicle $k$. Figure 2 shows how to calculate the path loss index using vehicle-to-vehicle communication.

Table 1. Parameters for vehicle signal strength.

\begin{tabular}{cccc}
\hline Pedestrian state & $\hat{P}(0)$ & $Q$ & $R$ \\
\hline Stationary & 1000 & 4.0210 & 26.8549 \\
Moving & 1000 & 3.8871 & 27.8656 \\
\hline
\end{tabular}

Table 2. Parameters for beacon signal strength.

\begin{tabular}{cccc}
\hline Pedestrian state & $\hat{P}(0)$ & $Q$ & $R$ \\
\hline Stationary & 1000 & 0.0129 & 22.5865 \\
Moving & 1000 & 3.0541 & 21.2856
\end{tabular}

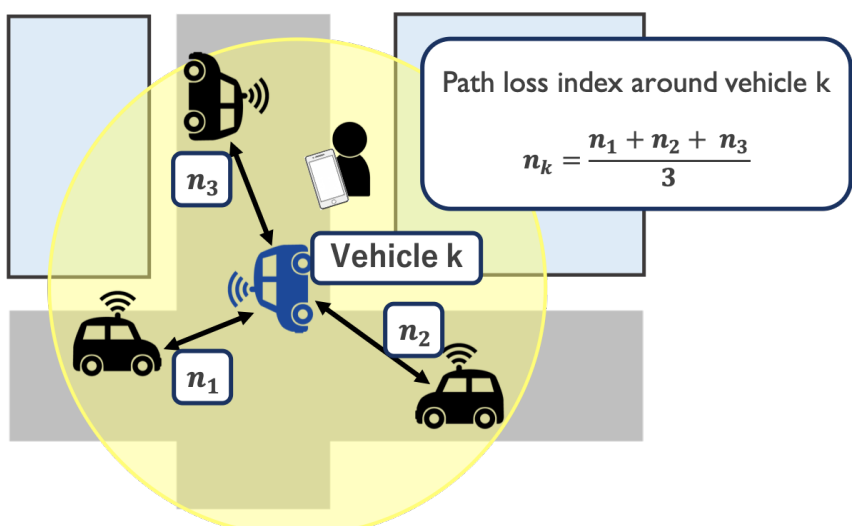

Figure 2. Calculation of dynamic path loss index. 


$$
\begin{gathered}
n_{i}=\frac{A-P\left(d_{i}\right)}{10 \log _{10} d_{i}} \\
n_{k}=\frac{\sum_{i=1}^{m} n_{i}}{m}(i \neq k)
\end{gathered}
$$

\subsection{Positioning Algorithm}

\subsubsection{Weighted Centroid Localization}

The position of the pedestrian is obtained using WCL, as expressed by Equation (3), Equation (4). The reason we use WCL in our proposed method is that the estimated position by WCL is less affected by the distance error than the least squares method. Regarding the path loss index, $n=2.0$ is used to determine the distance to the beacon, and the dynamic value in the previous section is used to determine the distance to the vehicle.

\subsubsection{Filtering Nodes}

As shown in Figure 3, WCL is useful when the target is inside the area where each source node of the signal is connected by the dashed line. However, the vehicles move and the number of them around the pedestrian changes over time. As a result, the positions of nodes tend to be biased. Since WCL calculates the weighted average of the positions of nodes, bias in the positions leads to a large error in the positioning result. Therefore, our proposed method selects the nodes suitable for WCL by filtering nodes, as shown in Figure 4. The detailed procedure of the algorithm is described below and Figure 5 shows the flow chart of the entire positioning algorithm, including the steps of filtering nodes.

i) Divide the coordinate space into four quadrants with the pedestrian position as the origin.

ii) Take the node closest to the pedestrian from each quadrant.

iii) Take the next closest node from each quadrant.

iv) Repeat step (iii) until there is a quadrant in which no node can be selected.

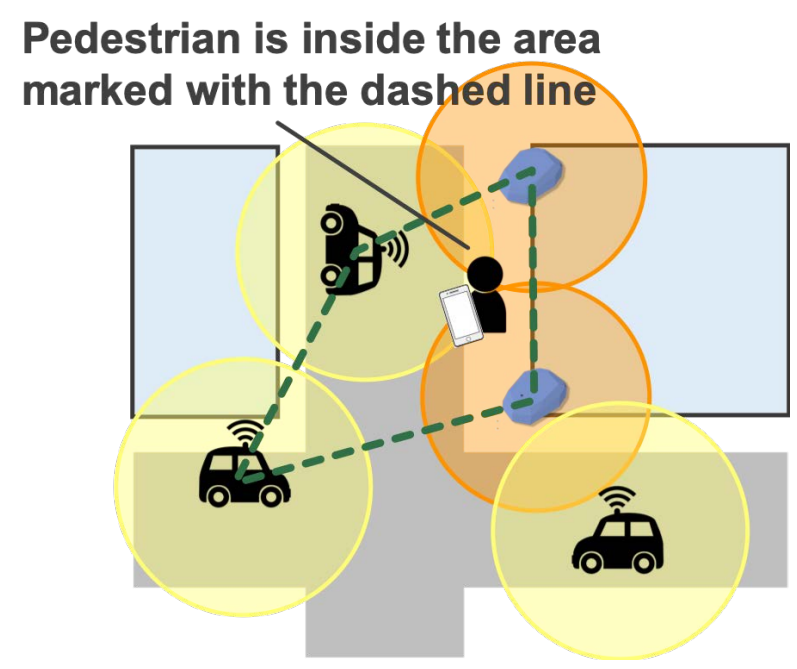

Figure 3. Situation where WCL is useful. 


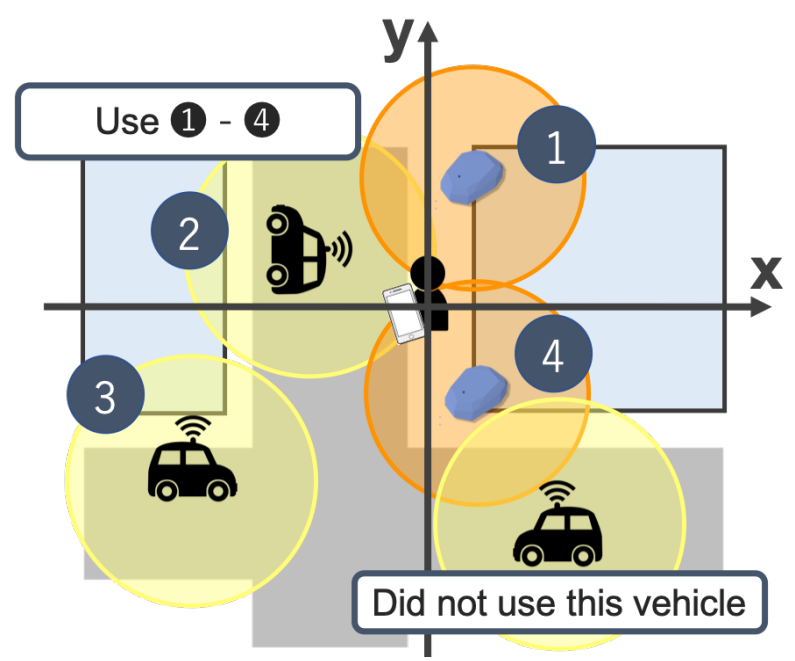

Figure 4. Information of filtered nodes.

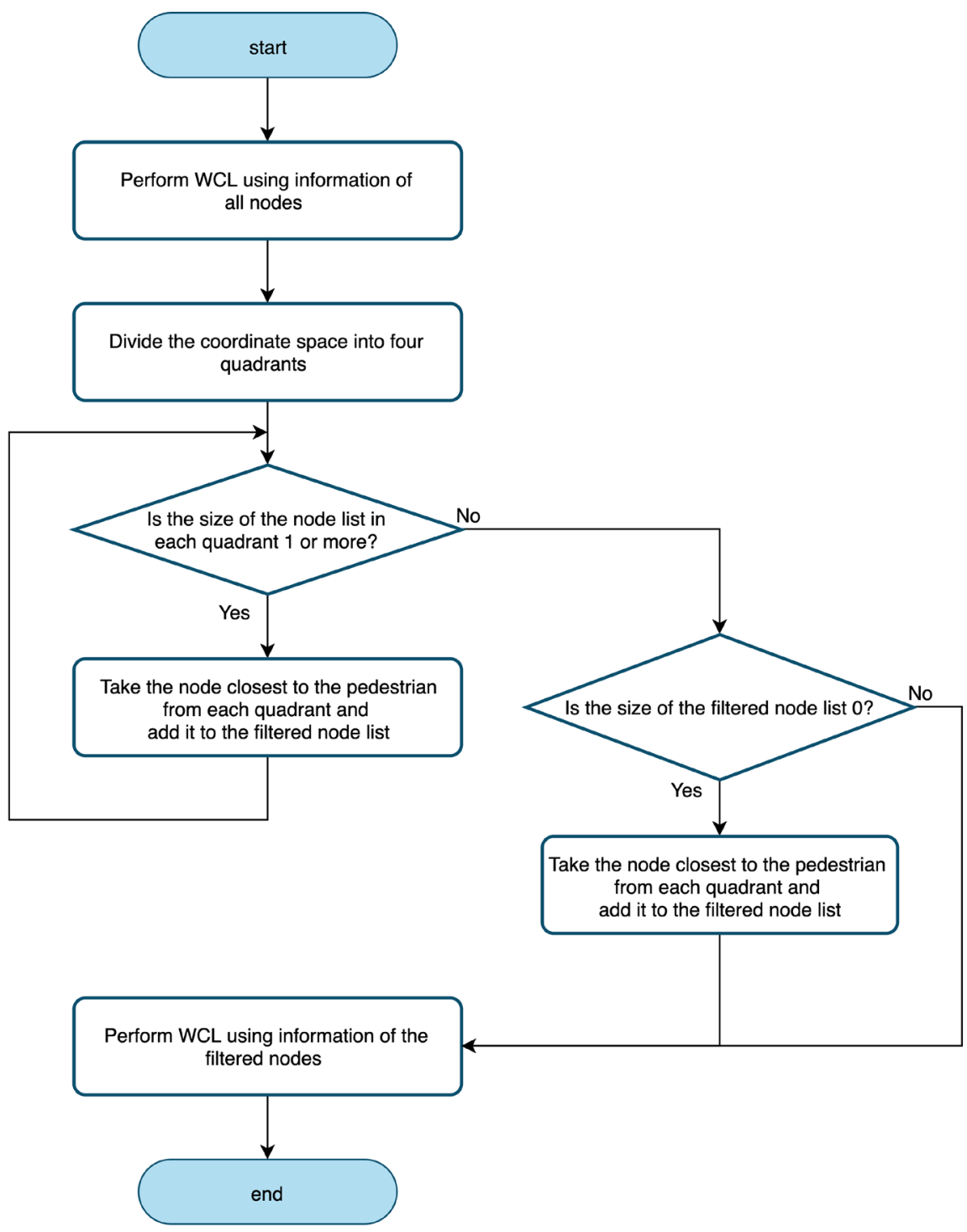

Figure 5. Flow chart of positioning algorithm. 
When there are no more nodes that can be selected from a quadrant, if one or more nodes have already been selected from each quadrant, we discard the last selected node from the first quadrant to the previous quadrant where no node can be selected. The WCL result using information of all nodes is the origin in step (i). The result of the second WCL using information of the filtered nodes is the final pedestrian position.

\section{Evaluation and Consideration}

\subsection{Simulation Environment}

We used Scenargie 2.1 [14], a network simulator developed by Space-Time Engineering (STE) to evaluate our proposed method. Figure 6 shows the simulation environment. This study aims to improve the positioning accuracy of pedestrians in urban areas, so we prepared an environment where pedestrians are surrounded by buildings. The white parts between the roads and the buildings are the sidewalks, and the pedestrian is on the sidewalk. We run simulations at each pedestrian point shown in Figure 6 and evaluated their average positioning error.

Table 3 shows the parameters used in the simulation. Mobility model GIS-BASED-RANDOM-WAYPOINT is a model in which each vehicle randomly determines a passing point and moves along the road to pass that point. ITU-R P.1411 [15] is a radio wave propagation model that considers road map information. Since the radio waves attenuate according to the shape of the road, this model is more realistic than a model that considers only the waves received directly and the waves reflected from the ground. For WCL, $g=3$ was used to obtain the pedestrian's initial position before filtering nodes, and $g=2$ was used to obtain the final position after filtering nodes. This is because information before filtering nodes contains more information with larger distance errors than information after filtering nodes.

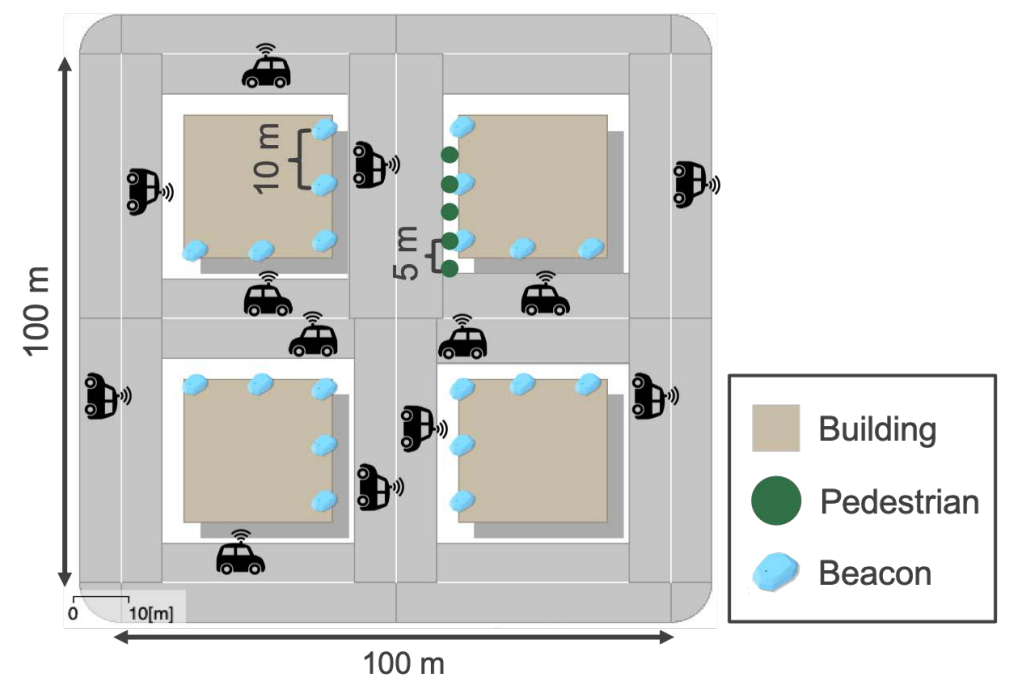

Figure 6. Simulation environment. 
Table 3. Simulation parameters.

\begin{tabular}{|c|c|c|}
\hline Simulator & \multicolumn{2}{|c|}{ Scenargie2.1 } \\
\hline Simulation time & \multicolumn{2}{|c|}{$20 \mathrm{~s}$} \\
\hline Beacon interval & \multicolumn{2}{|c|}{$10 \mathrm{~m}$} \\
\hline Number of vehicles & \multicolumn{2}{|c|}{80} \\
\hline Vehicle mobility model & \multicolumn{2}{|c|}{ GIS-BASED-RAMDOM-WAYPOINT } \\
\hline Vehicle speed & \multicolumn{2}{|c|}{$50 \mathrm{~km} / \mathrm{h}$} \\
\hline Vehicle position error & \multicolumn{2}{|c|}{$0 \mathrm{~m}$} \\
\hline Pedestrian speed & \multicolumn{2}{|c|}{$0 \mathrm{~m} / \mathrm{s}$} \\
\hline \multirow[t]{2}{*}{ Positioning interval } & \multicolumn{2}{|c|}{$500 \mathrm{~ms}$} \\
\hline & Beacon & Vehicle \\
\hline Frequency bands & $2.4 \mathrm{GHz}$ & $760 \mathrm{MHz}$ \\
\hline Communication intervals & $500 \mathrm{~ms}$ & $100 \mathrm{~ms}$ \\
\hline Transmission power & $-21 \mathrm{dBm}$ & $19.2 \mathrm{dBm}$ \\
\hline Pth & \multicolumn{2}{|c|}{$-50 \mathrm{dBm}$} \\
\hline Propagation model & \multicolumn{2}{|c|}{ ITU-R P.1411 } \\
\hline Fading model & \multicolumn{2}{|c|}{ Rayleigh } \\
\hline
\end{tabular}

\subsection{Evaluation}

To evaluate our proposed method, we compare the positioning error with those of existing methods. The existing methods to be compared are as follows:

- GPS

- Least squares method 1 (LS1)

The positioning algorithm is a least squares method. Filtering signal strengths, dynamic path loss index, and filtering nodes are not applied.

- Least squares method 2 (LS2)

The positioning algorithm is a least squares method. Filtering signal strengths and dynamic path loss index are applied.

- WCL1

The positioning algorithm is WCL. Filtering signal strengths, dynamic path loss index, and filtering nodes are not applied.

- WCL2

The positioning algorithm is WCL. Filtering signal strengths and dynamic path loss index are applied.

We also evaluate the effects of changing the number of vehicles, vehicle speed, and vehicle position error on our proposed method.

\subsection{Results and Consideration}

\subsubsection{Comparison of Positioning Errors with Those of Existing Methods} The simulation results are shown in Figure 7, Figure 8, and Table 4. The number of vehicles was 80 , all vehicle speeds were $50 \mathrm{~km} / \mathrm{h}$, and no error was included in the positions of all vehicles. Since it was difficult to measure the GPS 


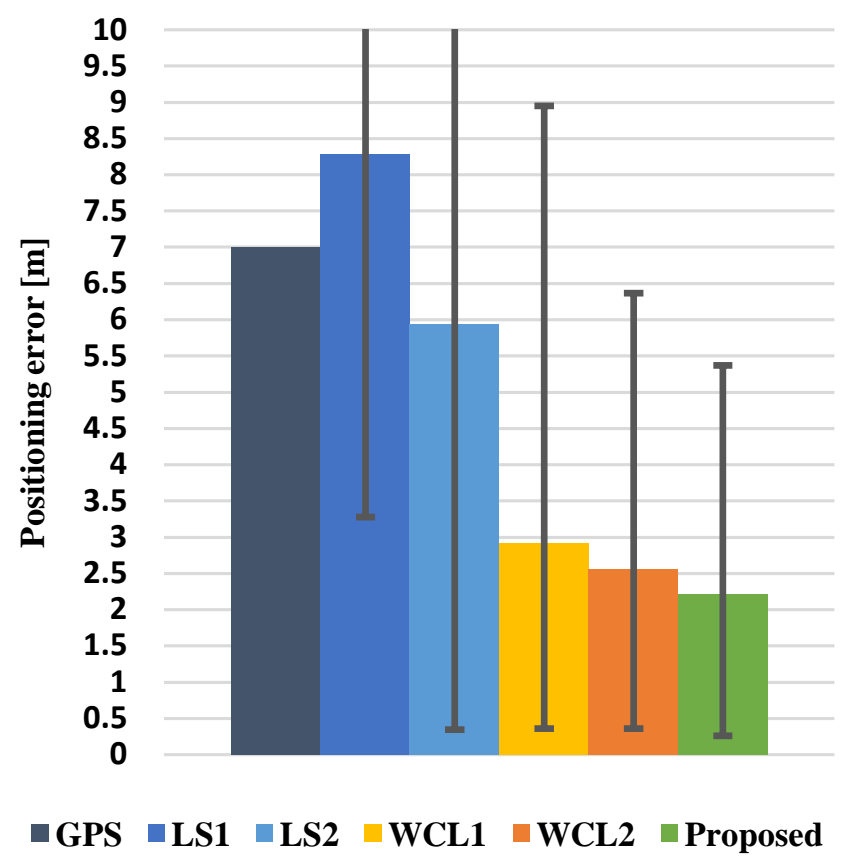

Figure 7. Positioning errors of each method.

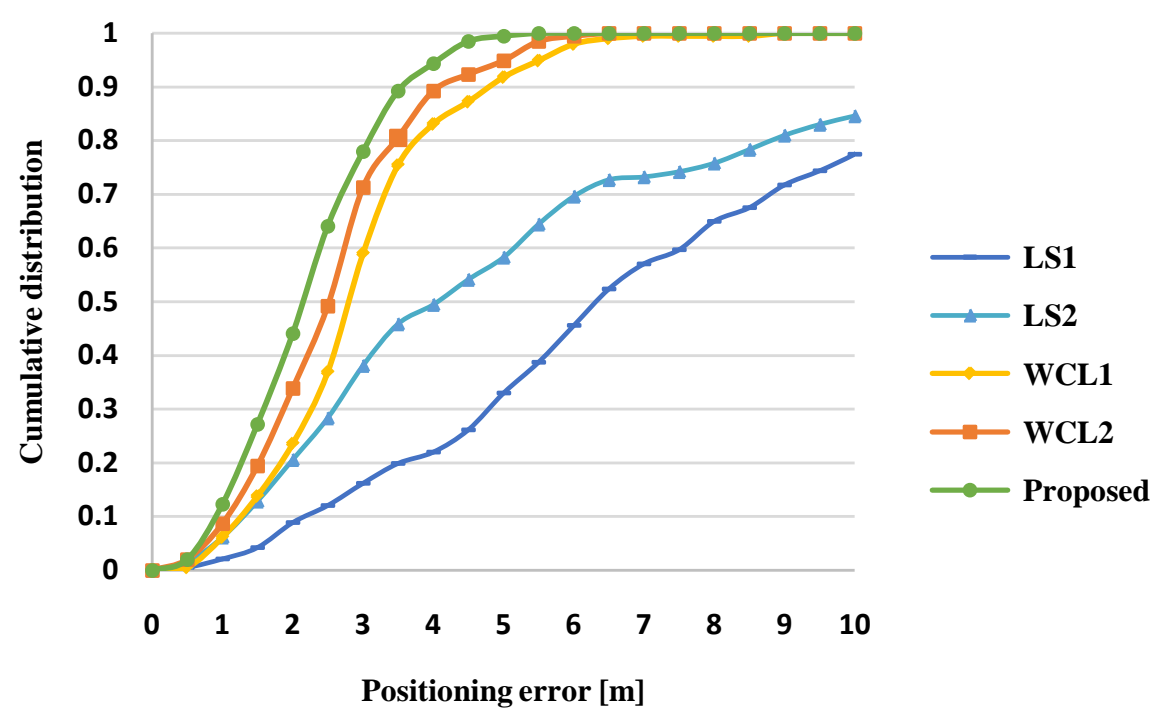

Figure 8. Cumulative distribution of positioning errors.

Table 4. Typical values for positioning errors.

\begin{tabular}{ccccc}
\hline & Average $[\mathrm{m}]$ & Standard deviation $[\mathrm{m}]$ & Max $[\mathrm{m}]$ & Min $[\mathrm{m}]$ \\
\hline GPS & 7 & - & - & - \\
LS1 & 8.28 & 8.74 & 94.56 & 0.39 \\
LS2 & 5.94 & 5.96 & 38.52 & 0.34 \\
WCL1 & 2.92 & 1.35 & 8.95 & 0.36 \\
WCL2 & 2.56 & 1.18 & 6.37 & 0.36 \\
Proposed & 2.21 & 1.02 & 5.37 & 0.26 \\
\hline
\end{tabular}


error on the simulator, we set it to $7 \mathrm{~m}$ based on the study of [3]. The values in Table 4 are rounded to the third decimal place. The results show that our method is more effective than the existing methods because the average, maximum, and minimum positioning errors obtained by our method are the smallest, and the convergence speed is also the fastest. Also, our method shows better results than WCL2, which means that filtering nodes enables more accurate and more stable position estimation. In addition, the positioning error of LS2 is smaller than that of LS1. Similarly, the error of WCL2 is smaller than that of WCL1. These results demonstrate that the filtering signal strengths and the use of dynamic path loss index are effective. In fact, the average distance errors were reduced from $2.38 \mathrm{~m}$ to $1.30 \mathrm{~m}$ for beacons and from $14.59 \mathrm{~m}$ to $10.64 \mathrm{~m}$ for vehicles.

\subsubsection{Effect of Number of Vehicles}

Figure 9 shows the simulation result with varying number of vehicles. All the vehicle speeds were set to $50 \mathrm{~km} / \mathrm{h}$, and there were no errors in the positions of all the vehicles. Since the number of nodes that can be used for positioning increase as the number of vehicles increases, the positioning error decreases. However, it remains flat at 80 or more vehicles. Therefore, 80 or more vehicles are necessary for stable positioning in the simulation environment. There are several reasons the error does not decrease with more than 80 vehicles. One is that the distance error remains even in our proposed method. It was shown in the previous section that the distance error can be reduced by our method, but the error cannot be eliminated completely, resulting in the errors in the estimation results. If the simulation is performed with the parameters in Table 3 assuming that there are no errors in the estimated distances from the pedestrian to each node, the average positioning error is about $1.74 \mathrm{~m}$ and the error is about $0.5 \mathrm{~m}$ smaller.

Another reason is the value of $g$ in WCL. The estimated position also depends

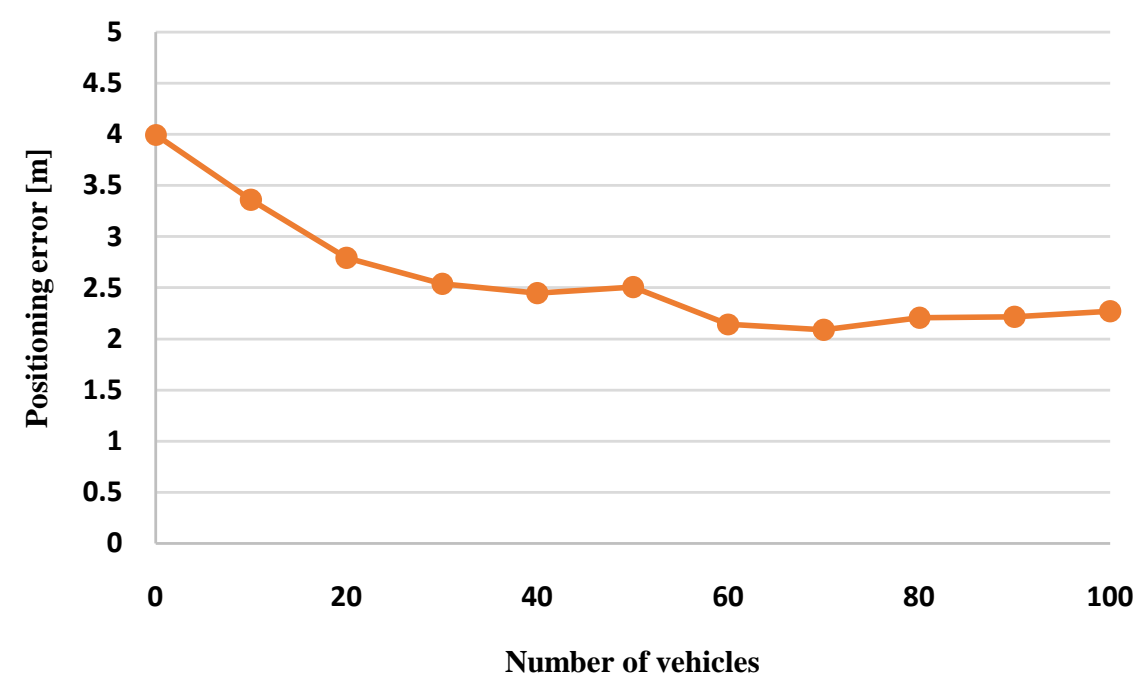

Figure 9. Changes in pedestrian's positioning error with increasing number of vehicles. 
on it. As shown in [11], there is a value of $g$ that can minimize the positioning error in a certain arrangement of nodes. However, because our proposed method uses vehicles as nodes, the arrangement of nodes changes over time. Therefore, to improve the positioning accuracy in the future, it is necessary to determine the value of $g$ dynamically in consideration of the node arrangement.

In addition, nodes filtered by our method are not always optimal. We divided the coordinate space into east-west and north-south, but depending on the arrangement of the nodes, it would be more effective to divide it at different angles, which is also our future task.

\subsubsection{Effect of Vehicle Speed}

Figure 10 shows the result of simulations with varying vehicle speed. There are 80 vehicles and no errors in the positions of all the vehicles. The maximum positioning error is about $2.46 \mathrm{~m}$ in $40 \mathrm{~km} / \mathrm{h}$ and the minimum positioning error is about $2.13 \mathrm{~m}$ in $20 \mathrm{~km} / \mathrm{h}$, but there is no significant difference in the change of the error with increasing vehicle speed. Therefore, the proposed method can ignore the effect of vehicle speed.

\subsubsection{Effect of Vehicle Position Error}

Figure 11 shows the result of simulations with 80 vehicles and all the vehicle speeds set to $50 \mathrm{~km} / \mathrm{h}$. Each vehicle position contains an error given in a uniform distribution. For example, when the error is $1 \mathrm{~m}$, an error of $1 \sqrt{2} \mathrm{~m}$ is given by a uniform distribution in both the horizontal and vertical position. As shown in this figure, in our proposed method, the positioning error of the pedestrian increases as the position error of the vehicle increases. However, when the vehicle position error is $3 \mathrm{~m}$ or less, the maximum change in the pedestrian error is 0.04 m. According to two studies [5] [6], the vehicle position error is at most $3 \mathrm{~m}$, so it is within the negligible range in our proposed method.

\section{Conclusion}

The GPS, which is currently most widely used as a method for acquiring position

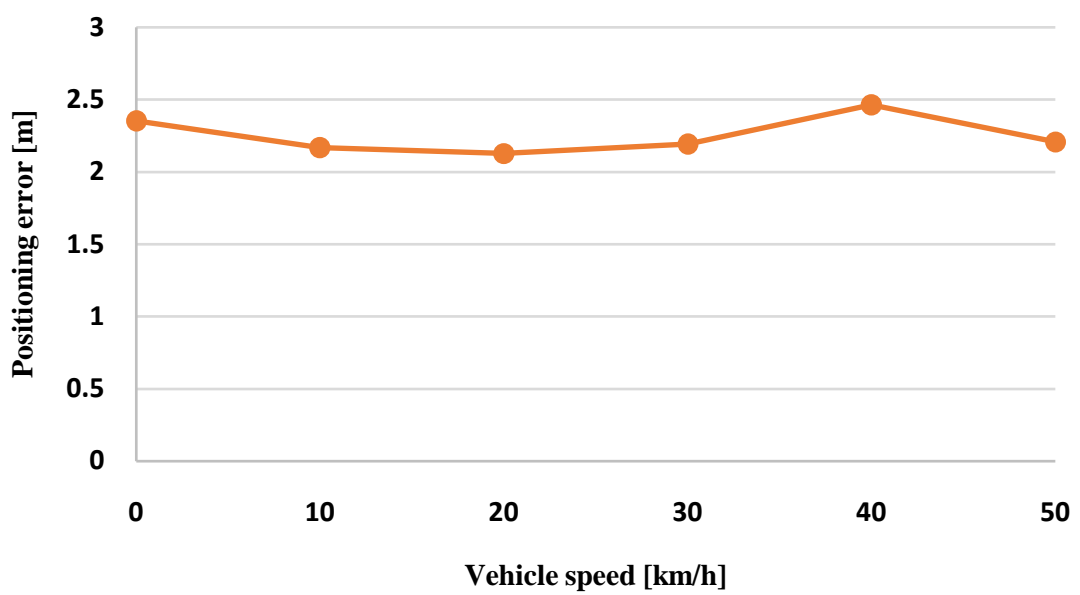

Figure 10. Changes in pedestrian's positioning error with increasing vehicle speed. 


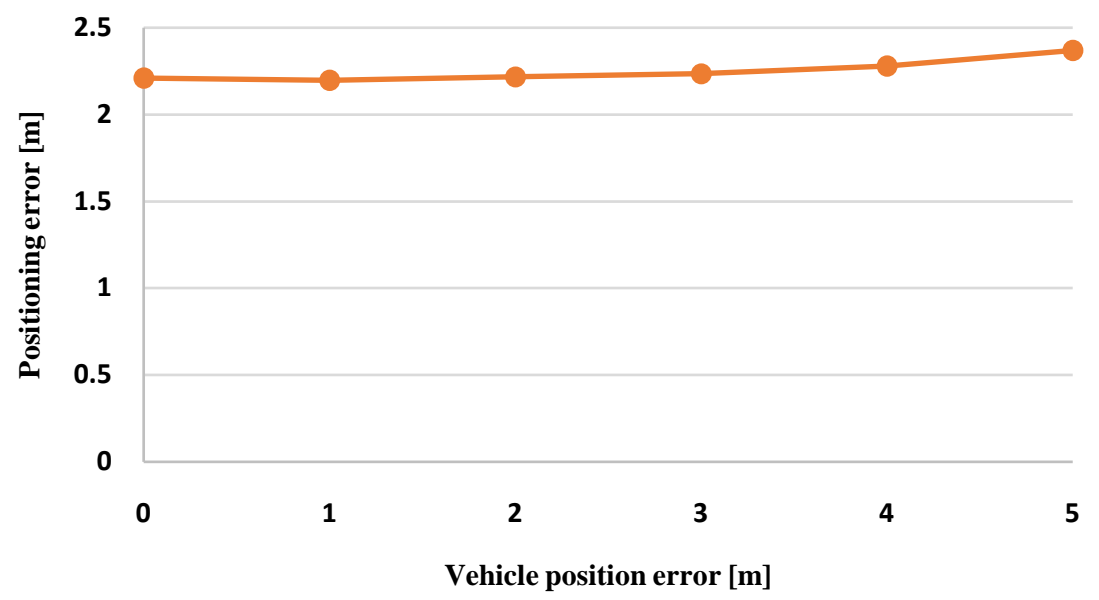

Figure 11. Changes in pedestrian's positioning error with increasing vehicle position error.

information, has a problem that the positioning error increases in urban areas. Therefore, to improve the positioning accuracy, we proposed a method using the signal strengths from vehicles and beacons. When using the signal strength, we faced the problems that the signal strength varies with time and how the path loss index is set. Our proposed method reduced the effect of these problems by applying the Kalman filter to reduce the fluctuation of the signal strength and by calculating the path loss index using vehicle-to-vehicle communication to dynamically cope with the surrounding radio wave propagation environment. We also aimed to further improve the accuracy by using WCL after filtering nodes. The positioning error was compared with those of existing methods using a simulator, and the evaluation results demonstrated that our method could reduce the positioning error. We also performed a simulation under various conditions. As a result, more than 80 vehicles were required in the simulation environment, and the effects of vehicle speed and vehicle position error could be ignored in our proposed method.

\section{Conflicts of Interest}

The authors declare no conflicts of interest regarding the publication of this paper.

\section{References}

[1] Anaya, J.J., Merdrignac, P., Shagdar, O., Nashashibi, F. and Naranjo, J.E. (2014) Vehicle to Pedestrian Communications for Protection of Vulnerable Road Users. IEEE Intelligent Vehicles Symposium Proceedings, Dearborn, 8-11 June 2014, 1037-1042. https://doi.org/10.1109/IVS.2014.6856553

[2] Sepulcre, M. and Gozalvez, J. (2012) Experimental Evaluation of Cooperative Active Safety Applications Based on V2V Communications. In: Proceedings of the 9 th ACM International Workshop on Vehicular Inter-Networking, Systems, and Applications, ACM, New York, 13-20. https://doi.org/10.1145/2307888.2307893

[3] Krista, M. and Pete, B. (2019) Smartphone GPS Accuracy Study in an Urban Envi- 
ronment. PLoS ONE, 14, e0219890. https://doi.org/10.1371/journal.pone.0219890

[4] Garnett, R. and Stewart, R. (2015) Comparison of GPS Units and Mobile Apple GPS Capabilities in an Urban Landscape. Cartography and Geographic Information Science, 42, 1-8. https://doi.org/10.1080/15230406.2014.974074

[5] Gu, Y., Hsu, L.-T. and Kamijo, S. (2015) Passive Sensor Integration for Vehicle Self-Localization in Urban Traffic Environment. Sensors (Basel), 15, 30199-30220. https://doi.org/10.3390/s151229795

[6] Suhr, J.K., Jang, J., Min, D. and Jung, H.G. (2017) Sensor Fusion-Based Low-Cost Vehicle Localization System for Complex Urban Environments. IEEE Transactions on Intelligent Transportation Systems, 18, 1078-1086. https://doi.org/10.1109/TITS.2016.2595618

[7] Xue, W., Qiu, W., Hua, X. and Yu, K. (2017) Improved Wi-Fi RSSI Measurement for Indoor Localization. IEEE Sensors Journal, 17, 2224-2230. https://doi.org/10.1109/ISEN.2017.2660522

[8] Varshney, V., Goel, R.K. and Qadeer, M.A. (2016) Indoor Positioning System Using Wi-Fi \& Bluetooth Low Energy Technology. 13th International Conference on Wireless and Optical Communications Networks, Hyderabad, 21-23 July 2016, 1-6. https://doi.org/10.1109/WOCN.2016.7759023

[9] Zhu, J.Y., et al. (2014) RSSI Based Bluetooth Low Energy Indoor Positioning. International Conference on Indoor Positioning and Indoor Navigation, Busan, 27-30 October 2014, 526-533. https://doi.org/10.1109/IPIN.2014.7275525

[10] Li, G., Geng, E., et al. (2018) Indoor Positioning Algorithm Based on the Improved RSSI Distance Model. Sensors, 18, 2820. https://doi.org/10.3390/s18092820

[11] Blumenthal, J., Grossmann, R., Golatowski, F. and Timmermann, D. (2007) Weighted Centroid Localization in Zigbee-Based Sensor Networks. IEEE International Symposium on Intelligent Signal Processing, Alcala de Henares, 3-5 October 2007, 1-6. https://doi.org/10.1109/WISP.2007.4447528

[12] Subedi, S., Kwon, G.-R., Shin, S., seung Hwang, S. and Pyun, J.-Y. (2016) Beacon Based Indoor Positioning System Using Weighted Centroid Localization Approach. Eighth Inter-National Conference on Ubiquitous and Future Networks, Vienna, 5-8 July 2016, 1016-1019. https://doi.org/10.1109/ICUFN.2016.7536951

[13] Tang, S. and Obana, S. (2018) Improving Performance of Pedestrian Positioning by Using Vehicular Communication Signals. IET Intelligent Transport Systems, 12, 366-374. https://doi.org/10.1049/iet-its.2017.0134

[14] Space-Time Engineering, Scenargie. https://www.spacetime-eng.com/jp

[15] P Series, Propagation Data and Prediction Methods for the Planning of Short-Range Outdoor Radiocommunication Systems and Radio Local Area Networks in the Frequency Range $300 \mathrm{MHz}$ to $100 \mathrm{GHz}$.

https://www.itu.int/dms pubrec/itu-r/rec/p/R-REC-P.1411-9-201706-I!!PDF-E.pdf 